\title{
Effect of Melatonin and Tetrapeptide on Gene Expression in Mouse Brain
}

\author{
S. V. Anisimov*,**, V. Kh. Khavinson ${ }^{* *}$, and V. N. Anisimov**,*** \\ Bulletin of Experimental Biology and Medicine, 2004, 138 (11): 570-576.
}

A microchip technique was used to study expression of 16,897 clones from a cDNA library in the brain of mice receiving melatonin or tetrapeptide Epithalon (Ala-Glu-AspGly). Expression of 53 transcripts in mouse brain underwent significant changes after treatment with the preparations. Melatonin and Epithalon modified expression of 38 and 22 transcripts, respectively. These preparations produced similar changes in the expression of 6 transcripts. Expression of 1 transcript (Rp119) was inhibited by melatonin, but induced by Epithalon. The target genes are physiologically related to the cell cycle, apoptosis, biosynthesis, processing, and transport of nucleic acids. Comparative study of gene expression in the brain and heart of CBA mice receiving melatonin and Epithalon suggest that these preparations have a tissue-specific biological effect.

Key Words: melatonin; peptide; Epithalon; gene expression; cDNA microchips

* Wallenberg Neuroscience Center, Lund University, Sweden;

** St. Petersburg Institute of Bioregulation and Gerontology, Northwestern Division of the Russian Academy of Medical Sciences;

*** N. N. Petrov Institute of Oncology, Russian Ministry of Health, St. Petersburg.

Address for correspondence: aging@mail.ru.

The pineal gland hormone melatonin plays an important role in physiological processes. Melatonin has in vitro and in vivo protective effects on neurons. This hormone protects various subtypes of neurons and whole brain from damages produced by ischemia, $\gamma$-irradiation, or neurotoxic agents [3,9,11]. Melatonin induces expression of antioxidant enzyme RNA and proteins [3]. Melatonin serves a free radical-trapping agent and is used for the therapy of patients with Alzheimer's disease, Parkinson's disease, and consequences of stroke or exposure of nerve cells to neurotoxins and hyperbaric oxygenation [3]. Melatonin is a major mediator of endocrine function in the pineal gland. It should be emphasized that activity of the pineal gland is realized via secretion of epiphyseal peptides [4]. Epithalamin, a complex peptide bioregulator of the pineal gland, decelerates aging, increases lifespan in drosophilae, mice and rats, and inhibits spontaneous and induced carcinogenesis in rodents [4]. The study of the amino acid composition in Epithalamin allowed us to synthesize Epithalon (Ala-Glu-Asp-Gly) with a molecular weight of $390.35 \mathrm{Da}$ [4]. This peptide has high geroprotective activity and suppresses growth of spontaneous and induced tumors in mice and rats. Fine mechanisms of the action of short peptides remain unclear. The effects of melatonin and Epithalon on the expression of 15,247 transcripts in the heart of CBA mice were evaluated by means of a microchip technique with cDNA macromatrices $[1,2]$. The primary study of target 
genes for melatonin and peptides would elucidate the mechanism for their biological action in tissues. Here we studied the effects of melatonin and Epithalon on expression of 16,897 transcripts in mouse brain.

\section{MATERIALS AND METHODS}

Experiments were performed on 30 female CBA mice obtained from the Rappolovo nursery (Russian Academy of Medical Sciences). The animals received ad libitum food and water and were kept at a 12:12-h light/dark regimen. At the age of 6 months the mice were divided into 3 groups of 10 specimens each. Control animals received subcutaneous injections of $0.1 \mathrm{ml}$ physiological saline. Epithalon was synthesized by E. I. Grigor'ev at the St. Petersburg Institute of Bioregulation and Gerontology. This preparation was dissolved in $0.1 \mathrm{ml}$ physiological saline and injected subcutaneously in a dose of $0.1 \mu \mathrm{g}$. Melatonin $(20 \mathrm{mg} /$ liter, Sigma) was given ad libitum with water for 1 night (18.00-9.00). The mice were decapitated on day 6 . The brain was removed, immediately frozen in liquid nitrogen, and stored at $-80^{\circ} \mathrm{C}$ until total RNA isolation.

Experiments were performed with the library of clones NIA 17K (cDNA collection of the US National Institute of Aging, NIA). The reformatted combination of NIA 15K [7] and NIA 7,4K libraries [13] consisted of 16,897 clones (more than 12,000 unique gene products). Clones were placed on 2 nylon membranes marked by letters $\mathrm{A}$ and B. The distance between centers of spots was $0.85 \mathrm{~mm}$. The diameter of spots was $0.4 \mathrm{~mm}$. The information about nucleotide sequences of clones is available at the site of the Laboratory of Genetics (US National Institute of Aging, http://www.lgsun.grc.nia.nih.gov/). Detailed description of the technique to obtain cDNA macromatrices is presented at the site of the Department of Microchips (Johns Hopkins University, US National Institute of Aging; (http://www.daf.jhmi.edu/microarray/index.htm).

The samples were prepared, treated with isotopes, and hybridized with cDNA macromatrices as described elsewhere [1,2]. Total RNA was isolated by the guanidine isothiocyanate method [1] and used as the substrate $(20 \mu \mathrm{g})$ to introduce a radioactive isotope of phosphorus 33P (Amersham) during cDNA synthesis with SuperScript II reverse transcriptase (Life Technologies) on the oligo(dT)12-18 primer (Amersham). Each group was exposed to 2 independent hybridizations. Experiments were performed with independently synthesized samples. After normalization by total emission, the samples were hybridized with prehybridized membranes of macromatrices using the mixture of MicroHyb (Research Genetics), Cot1 (1 $\mu \mathrm{g} / \mathrm{ml}$, Life Technologies), and Poly(A) $\left(50 \mu \mathrm{g} / \mathrm{ml}\right.$, Amersham) at $42^{\circ} \mathrm{C}$ for $18 \mathrm{~h}$. Washed membranes were exposed to a Storage Phosphor screen (Molecular Dynamics) for 5 days and scanned with a Storm 860 automatic radiation detector (resolution $50 \mu$ pixels, Amersham). The formatted image was analyzed by means of Array-Pro Analyzer 4.0.0.41 software (Media Cybernetics). Coordinate grids were superimposed on each image to estimate the strength of a signal from individual clones (arb. units). Background signals were locally normalized. The results of hybridization were normalized by the method of Z-transformation. The difference between common logarithms of signal strength and average signal strength was divided by the standard deviation of the common logarithm of the signal. Relative expression was estimated by comparing the average results of experimental and control 
hybridization. The difference between Z-transformations of experimental and control groups was divided by the standard deviation to calculate the Z-ratio $\left(Z_{\text {rat }}\right)[1,2]$. The coefficient of variation was estimated for each membrane. We calculated the ratio between the standard deviation of the common logarithm of the signal and mean value of the common logarithm of the signal (\%). The correlation coefficient was determined for each set of clones (MS Excel XP software).

\section{RESULTS}

33P-Labeled samples (2 samples of each group) were synthesized from independently isolated samples of mouse brain total RNA and hybridized with cDNA macromatrices consisting of 16,897 DNA clones (Fig. 1). Screening with 16,897 clones revealed significant expression of $65.7,62.7$, and $59.8 \%$ clones in control animals and mice receiving Epithalon and melatonin, respectively ( $Z$-transformation $>0)$. In these mice the ratio of highly expressed clones was 8.2, 9.4, and $10.5 \%$, respectively (Ztransformation $>1$ ). The coefficients of variation and correlation confirmed high accuracy of hybridization. The correlation coefficients for hybridization of samples from control animals and mice receiving Epithalon and melatonin were 0.995, 0.991, and 0.984, respectively. The average coefficients of variation were $36.0,33.6$, and $33.8 \%$, respectively.

The amount of gene products characterized by significant changes in the level of expression was extremely low (53 clones, $0.31 \%$ of the total number, $1.5 \leq Z_{\text {rat }} \leq-1.5$ ). However, a considerable number of clones exhibited high level of expression (Fig. 1). In mice receiving melatonin and Epithalon, expression of clones increased (5 and 9 clones, respectively) or decreased (33 and 13 clones, respectively, Table 1). Study of normalized $Z_{\text {rat }}$ in animals receiving melatonin and Epithalon showed that maximum changes in the level of expression were +5.02 (RIKEN cDNA 1810049E02 gene) and -5.10 (male germ cell-associated kinase, Mak), respectively. Nucleotide sequences of 11 transcripts with modified expression (20.8\%) corresponded to the known genes. Six clones did not exhibit similarity to the GenBank genetic information, since study of nucleotide sequences in the mouse genome was uncompleted. Nucleotide sequences of 36

transcripts corresponded to expressed gene fragments (ESTs) that were similar (7 transcripts) or dissimilar to the known genes (22 transcripts). The known genes belonged to various functional classes, including cell systems for signaling and communication (transforming growth factor $\beta 1$-induced transcript 4 , guanine nucleotide-releasing factor RAN (Mog1), and ESTs similar to isoform 1 of human caldesmon gene), expression of genes and proteins (ribosomal protein L19 (Rp19), ribonucleic acid-binding protein S1, and interferon regulatory factor 3 (Irf3)) and metabolism (Tousled-like kinase 2 (Tlk2), dihydrolipoamide branched chain transacylase E2 (Dbt), and guanosine monophosphate reductase isologue (Gmpr2)). Screening procedure did not reveal significant changes in expression of genes for glutathione peroxidases $(\mathrm{Gpx} 1,2,3,4)$ and superoxide dismutases (Sod1,2). Maximum values of $Z_{\text {rat }}$ for these genes in rats receiving melatonin and Epithalon were +0.33 (Gpx2) and +0.27 (Sod1), respectively. The databank of the US National Institute of Health for detection, visualization, and complex study (http://apps1.niaid.nih.gov/david) was used for further identification of transcripts similar to nucleotide sequences of clusters in the UniGene databank (46 transcripts). According to the classification of biological processes, 5 and 3 transcripts corresponded to 
categories of metabolism, cell growth, and/or maintenance of viability, respectively. Study with classification of molecular function revealed a correspondence of transcripts to categories of nucleic acid binding $(n=4)$ and transferase activity $(n=3)$.

Published data show that melatonin and Epithalon possess protective properties in the brain $[4,6]$. It can be hypothesized that several transcripts with modified expression are involved in the mechanism of action of these substances. Previous studies showed that melatonin inhibits expression of Tousled-like kinase 2 (Tlk2). This serine/threonine kinase is regulated by the cell cycle and functionally coupled to DNA replication [10]. Human Tlk genes affect chromatin assembly during DNA replication. Their activity is maximum in S-phase of the cell cycle and decreases under conditions of DNA damage and treatment with replication inhibitors [8]. Mutation-produced changes in Tlk activity are followed by arrest of cell division (interphase) and apoptosis. However, overexpression of Tlk impairs chromatin structure [8]. Melatonin inhibited expression of a guanine nucleotide-releasing factor RAN (Mog1). The mean values of $Z_{\text {rat }}$ for Tlk2 and Mog1 were -1.55 and -1.65 , respectively. The factor performs similar physiological functions and is required for nucleic protein import [3]. This factor is associated with RAN during DNA replication, regulation of the cell cycle, biosynthesis, processing, and transport [3]. The mean value of $Z_{\text {rat }}$ for transforming growth factor $\beta 1$-induced transcript 4 (Tsc22) in the brain of mice receiving melatonin was -1.60 . This factor plays an important role in the regulation of signals for cell death after DNA damage [5]. Overexpression of the factor initiates apoptosis in human tumor cells [12]. None of these genes were described in studying the biological effect of melatonin or epiphyseal peptides.

Melatonin significantly inhibited expression (average $Z_{\text {rat }}-2.12$ ) of the gene for interferon regulatory factor 3 (Irf3), which was probably related to the existence of functional relationship between melatonin, CREB-binding protein (CREBBP), and Irf3. The balance between phosphorylation and dephosphorylation of pCREB/CREB is mediated by serine/threonine phosphatase, modulates expression of the gene for arylalkylamine-N-acetyltransferase (Aanat), and regulates melatonin biosynthesis [14]. CREBBP and Irf3 contribute to coactivation of the mechanism mediated by doublestranded RNA-activated transcription factor 1 (DRAF1). Nuclear translocation of IRF3 is accompanied by the increase in phosphorylation of serine and threonine, activation of DRAF1, and specific changes in gene expression produced by viral infections [15].

Expression of 6 transcripts (dihydrolipoamide branched chain transacylase E2 (Dbt), 4 unidentified genes and expressed gene fragments (ESTs, expressed sequence tags), and mitochondrial gene for NADH dehydrogenase 4; mt-Nd4) underwent similar changes in mice receiving melatonin and Epithalon. Expression of 1 transcript (ribosomal L19 (Rp119)) was inhibited by melatonin, but induced by Epithalon. Mutations in the only described gene of this group (Dbt) are followed by the development of type 2 maple syrup urine disease (MSUD2). The molecular mechanisms underlying suppression of gene expression in the brain of mice receiving melatonin and Epithalon remain unclear.

Comparative study revealed qualitatively different changes in gene expression in the brain and heart of mice receiving melatonin and Epithalon [1,2]. The transcript corresponding to mouse mitochondrial gene for NADH dehydrogenase 4 (mt-Nd4) was the only transcript whose expression in the brain and heart of CBA mice underwent similar changes after treatment with Epithalon (average $Z_{\text {rat }} 2.31$ and 2.03, respectively) 
and melatonin (average $Z_{\text {rat }} 1.71$ and 1.90, respectively). Transcription of other transcripts in tissues did not significantly change. Our results confirm the hypothesis that Epithalon [4] and melatonin [3] have a tissue-specific biological effect. The action of melatonin can be mediated by specific receptors and various transcription factors. A screening procedure was performed with 16,897 and 15,247 transcripts in the brain and heart of mice, respectively. The number of highly expressed transcripts in the brain was much lower than in the heart. These differences can be related to the above mentioned reason. A larger sample of tissues should be studied to derive a final conclusion about the tissuespecific effect of Epithalon and melatonin.

A large-scale study of the influence of melatonin and Epithalon on gene expression in the brain of mice revealed their specific and general effects. Target genes are physiologically related to the cell cycle, apoptosis, biosynthesis, processing, and transport of nucleic acids.

This work was supported by the US National Institute of Aging and Russian Foundation for Basic Research (grant No. 00-04-48481).

We are grateful to K. Becker, V. Wood, H. Spurgen, M. Volkova, K. Boheler, and E. Lakatta (US National Institute of Aging, Baltimore) for their help in performing the study.

\section{REFERENCES}

1. S. V. Anisimov, K. R. Boheler, and V. N. Anisimov, Dokl. Ros. Akad. Nauk, 383, 90-95 (2002).

2. S. V. Anisimov, K. R. Boheler, V. Kh. Khavinson, and V. N. Anisimov, Byull. Eksp. Biol. Med., 133, No. 3, 340-347 (2002).

3. F. I. Komarov, S. I. Rapoport, N. K. Malinovskaya, and V. N. Anisimov, Melatonin under Normal and Pathological Conditions

[in Russian], Moscow (2004).

4. V. Kh. Khavinson and V. N. Anisimov, Peptide Bioregulators and Aging [in Russian], St. Petersburg (2003).

5. S. Hino, H. Kawamata, F. Omotehara, et al., Biochem. Biophys. Res. Commun., 292, 957-963 (2002).

6. C. H. Hsu, B. C. Chi, and J. E. Casida, J. Pineal Res., 32, 53-58 (2002).

7. G. J. Kargul, D. B. Dudekula, Y. Qian, et al., Nat. Genet., 28, 17-18 (2001). (2003).

8. D. R. Krause, J. C. Jonnalagadda, M. H. Gatei, et al., Oncogene, 22, 5927-5937

9. R. M. Sainz, J. C. Mayo, C. Rodriguez, et al., Cell. Mol. Life Sci., 60, 14071426 (2003).

10. H. H. Sillje, K. Takahashi, K. Tanaka, et al., EMBO J., 18, 5691-5702 (1999).

11. L. Thomas, J. E. Drew, D. R. Abramovich, and L. M. Williams, Int. J. Mol. Med., 1, 539-543 (1998).

12. D. Uchida, H. Kawamata, F. Omotehara, et al., Lab. Invest., 80, 955-963

13. V. VanBuren, Y. Piao, D. B. Dudekula, et al., Genome Res., 12, 1999-2003 (2002). 
14. C. von Gall, A. Lewy, C. Schomerus, et al., Eur. J. Neurosci., 12, 964-972 (2000).

15. B. K. Weaver, K. P. Kumar, and N. C. Reich, Mol. Cell. Biol., 18, 1359-1368 (1998). 


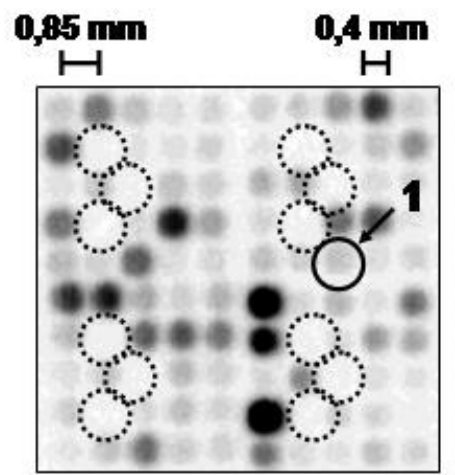

control

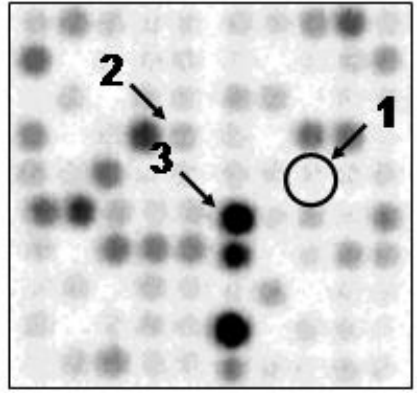

melatonin

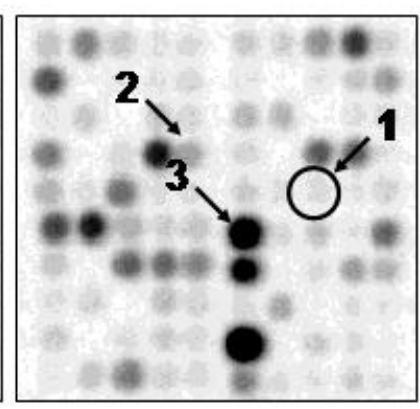

Epithalon

Fig. 1. Hybridization of brain samples from mice receiving physiological saline (control), melatonin, or Epithalon. Complete circle: clone with variations in expression, $\mathrm{Z}_{\text {rat }} \leq-1.5$ (melatonin, -1.69; Epithalon, -0.93). Dotted circles: non-cDNA-containing spots. 1) expressed gene fragments (ESTs), Mm.26176 cluster of UniGene databank; 2) guanosine triphosphate-binding protein 3 (Gtpbp3-pending,); 3) subunit 2 ofsplicing factor 3a (Sf3a2). Scales: diameter of membrane spots with applied cDNA $(0.4 \mathrm{~mm})$ and distance between adjacent spots $(0.85 \mathrm{~mm})$. 
TABLE 1. Effects of Melatonin and Epithalon on Gene Expression in Mouse Brain.

\begin{tabular}{|c|c|c|c|c|c|c|c|}
\hline \multirow{2}{*}{$\mathrm{N}$} & \multicolumn{2}{|c|}{ Microchip } & \multicolumn{2}{|c|}{$Z_{\text {Ratio }}$} & \multicolumn{2}{|c|}{ Databank } & \multirow{2}{*}{ Gene } \\
\hline & $\mathrm{A} / \mathrm{B}$ & Position & Melatonin & Epithalon & GenBank & UniGene & \\
\hline 1. & B & $44-2: 2$ & 5.02 & 3.96 & *BG085452 & Mm.28765 & RIKEN cDNA 1810049E02 gene \\
\hline 2. & $\mathrm{~B}$ & $175-3: 1$ & 1.90 & 2.31 & *AW551351 & No data & Mitochondrial gene for NADH dehydrogenase 4 (mt-Nd4) \\
\hline 3. & $\mathrm{~B}$ & $72-2: 3$ & 2.12 & 1.18 & BG073021 & Mm.28367 & Expressed AA408740 sequence \\
\hline 4. & A & $196-3: 4$ & 2.13 & 0.57 & BG066802 & Mm.172842 & ESTs weakly associated with retroviral polyprotein POL (POL2) \\
\hline 5. & $\mathrm{~B}$ & $117-2: 1$ & 0.29 & 2.03 & BG072397 & No data & No data \\
\hline 6. & $\mathrm{~B}$ & $36-2: 5$ & 1.51 & 0.63 & BG073331 & Mm.1951 & Ribonucleic acid-binding protein S1 \\
\hline 7. & $\mathrm{~B}$ & $69-2: 3$ & -0.31 & 1.81 & BG073008 & Mm.182624 & ESTs, weakly associated with RIKEN cDNA 5730493B19 gene \\
\hline 8. & $\mathrm{~B}$ & $19-2: 2$ & -1.62 & 2.55 & *BG085419 & Mm.30806 & Ribosomal protein L19 (Rp19) \\
\hline 9. & $\mathrm{~B}$ & $154-1: 4$ & 0.35 & -1.74 & BG071739 & Mm.104106 & ESTs \\
\hline 10. & $\mathrm{~B}$ & $95-1: 2$ & -1.51 & 0.04 & BG071020 & Mm.173350 & ESTs \\
\hline 11. & $\mathrm{~B}$ & $1-3: 5$ & -1.55 & 0.05 & BG087221 & Mm.4557 & Tousled_like kinase 2 (Tlk2) \\
\hline 12. & A & $185-1: 2$ & 1.10 & -2.61 & BG063398 & Mm.155099 & RIKEN cDNA B230219D22 gene \\
\hline 13. & A & $57-4: 5$ & -1.55 & -0.01 & AU022883 & Mm.182846 & ESTs \\
\hline 14. & $\mathrm{~B}$ & $117-4: 4$ & 1.25 & -2.86 & AW556794 & Mm.29346 & Human Gmpr2 gene for guanosine monophosphate reductase (LOC51292) \\
\hline 15. & $\mathrm{~B}$ & $247-4: 4$ & -1.52 & -0.17 & AW557098 & No data & No data \\
\hline 16. & $\mathrm{~B}$ & $30-1: 5$ & -0.08 & -1.64 & BG071980 & Mm.174051 & ESTs, moderately associated with reverse transcriptase \\
\hline 17. & $\mathrm{~B}$ & $25-1: 2$ & -1.62 & -0.10 & BG070878 & Mm.189628 & EST, chromosome 2 \\
\hline 18. & $\mathrm{~B}$ & $35-1: 3$ & -1.60 & -0.13 & BG084173 & Mm.20927 & Transforming growth factor $\beta 1$-induced transcript 4 \\
\hline 19. & A & $246-1: 2$ & -1.56 & -0.16 & BG063470 & Mm.182543 & ESTs \\
\hline 20. & A & $132-5: 3$ & 0.15 & -1.90 & BG082634 & No data & No data \\
\hline 21. & $\mathrm{~B}$ & $356-1: 3$ & -2.39 & 0.57 & BG071576 & Mm.173988 & ESTs \\
\hline 22. & $\mathrm{~B}$ & $287-5: 1$ & -2.06 & 0.15 & - & No data & No data \\
\hline 23. & A & $378-4: 5$ & -0.03 & -1.94 & BG068750 & Mm.173656 & ESTs \\
\hline 24. & $\mathrm{~B}$ & $161-2: 1$ & -1.91 & -0.07 & BG072440 & Mm.17993 & RIKEN cDNA 1700027N10 gene \\
\hline 25. & $\mathrm{~B}$ & $329-1: 4$ & -0.36 & -1.62 & BG071888 & Mm.17870 & $\begin{array}{l}\text { ESTs, moderately associated with COI protein } \\
\text { ESTs, strongly associated with cerebral cavernous malformation gene } 1\end{array}$ \\
\hline 26. & $\mathrm{~B}$ & $80-5: 5$ & -1.74 & -0.32 & BQ552303 & Mm.217895 & $(\mathrm{Ccm} 1)$ \\
\hline 27. & $\mathrm{~B}$ & $323-3: 1$ & -1.64 & -0.46 & BG086597 & Mm.205010 & RIKEN cDNA 2810031L11 gene \\
\hline 28. & $\mathrm{~B}$ & $205-5: 4$ & -1.58 & -0.53 & BQ551867 & Mm.221652 & ESTs \\
\hline 29. & A & $55-5: 1$ & -1.85 & -0.29 & BG068805 & Mm.26393 & RIKEN cDNA 4930533G20 gene \\
\hline 30. & $\mathrm{~B}$ & $303-5: 3$ & 1.46 & -3.66 & BQ551396 & Mm.221605 & ESTs \\
\hline
\end{tabular}




\begin{tabular}{|c|c|c|c|c|c|c|c|}
\hline \multirow{2}{*}{$\mathrm{N}$} & \multicolumn{2}{|c|}{ Microchip } & \multicolumn{2}{|c|}{$Z_{\text {Ratio }}$} & \multicolumn{2}{|c|}{ Databank } & \multirow{2}{*}{ Gene } \\
\hline & $\mathrm{A} / \mathrm{B}$ & Position & Melatonin & Epithalon & GenBank & UniGene & \\
\hline 31. & B & 297-5:3 & -1.52 & -0.71 & BQ551391 & Mm.217817 & ESTs \\
\hline 32. & B & $192-4: 4$ & -0.69 & -1.55 & BG088274 & Mm.30077 & $\begin{array}{l}\text { ESTs, weakly associated with isoform } 1 \text { of human caldesmon gene (L-CAD, } \\
\text { H-CAD, NAG22 protein, I-caldesmon I) }\end{array}$ \\
\hline 34. & A & $40-2: 5$ & -1.67 & -0.68 & BG065627 & Mm.24899 & ESTs \\
\hline 35. & B & $133-4: 4$ & -1.58 & -0.78 & BG088220 & Mm.18561 & Gene for cysteine- and histidine-rich protein 1 (Cyhr1) \\
\hline 36. & B & $301-5: 4$ & -1.68 & -0.67 & BQ552034 & Mm.221668 & ESTs \\
\hline 37. & A & $45-1: 5$ & -1.65 & -0.73 & BG077598 & Mm.143774 & Guanine nucleotide-releasing factor RAN (Mog1) \\
\hline 38. & B & $329-1: 3$ & -0.61 & -1.81 & AU043423 & No data & No data \\
\hline 39. & A & $275-5: 3$ & -2.12 & -0.34 & BG069754 & Mm.3960 & Interferon regulatory factor (Irf3) \\
\hline 40. & B & $25-3: 5$ & -1.88 & -0.63 & BG074613 & Mm.77786 & ESTs \\
\hline 41. & B & 174-4:4 & -2.03 & -0.52 & BG075723 & Mm.103003 & Expressed sequence AU015603 \\
\hline 42. & A & $28-5: 3$ & -1.69 & -0.93 & BG069529 & Mm.26176 & ESTs \\
\hline 43. & B & $227-4: 3$ & -1.64 & -1.05 & BG075441 & Mm.103168 & ESTs \\
\hline 44. & A & $48-1: 4$ & -1.35 & -1.85 & BG063926 & Mm.197220 & ESTs \\
\hline 45. & A & $141-2: 5$ & -2.20 & -1.04 & BG065708 & Mm.27654 & $\begin{array}{l}\text { ESTs, weakly associated with the gene for Diaphanous protein } \\
\text { homologue } 1 \text { (DIA1, DRF1, p140mDIA) }\end{array}$ \\
\hline 46. & A & $5-5: 2$ & -1.85 & -1.49 & BG069123 & Mm.173776 & ESTs \\
\hline 47. & B & $48-2: 1$ & -1.03 & -2.40 & BG072343 & Mm.11227 & Expressed sequence AU017169 \\
\hline 48. & A & 207-5:3 & -1.92 & -1.61 & *BG069687 & Mm.33532 & EST, chromosome 16 \\
\hline 49. & B & $211-1: 2$ & -3.28 & -0.61 & BG071133 & No data & No data \\
\hline 50. & A & 277-3:4 & -1.91 & -2.40 & *BG080013 & Mm.34798 & RIKEN cDNA 4930506D01 gene \\
\hline 51. & B & $151-4: 4$ & -2.59 & -1.99 & *BI076814 & Mm.3636 & Dihydrolipoamide branched chain transacylase E2 (Dbt) \\
\hline 52. & B & 270-4:4 & -2.74 & -2.45 & *AW557182 & Mm.43213 & RIKEN cDNA 9030402K04 gene \\
\hline 53. & A & $234-5: 2$ & -0.77 & -5.10 & BG082366 & Mm.8149 & Male germ cell associated kinase (Mak) \\
\hline
\end{tabular}

Note. Transcripts with significant changes in expression (1.5 $\leq$ Zrat $\leq-1.5)$. Transcripts were classified $(\mathrm{N})$ by mean values of $Z_{\text {rat }}$ in experimental groups. $A / B$ : membrane of microchip (A or B). Position: position of a clone in the membrane (grid). Z-ratios: mean values in experimental groups (1.5 $\leq Z_{\text {rat }} \leq-1.5$ : printed in italic type). GenBank: index of GenBank databank (NCBI). UniGene: number of a cluster in UniGene databank (NCBI). *Statistically significant changes in expression of transcripts in both experimental groups (melatonin and Epithalon). EST ( expressed sequence tag): expressed gene fragment. 